\title{
Experimental Test of a New Precision Model for Microwave Rotary Vane Attenuators
}

\section{Guldbrandsen, Tom; Guldbrandsen, Birthe; Warner, Frank L.; Herman, Peter; Parkes, Gordon W.;}

\section{Published in:}

I E E E Transactions on Instrumentation and Measurement

Link to article, DOI:

10.1109/TIM.1983.4315061

Publication date:

1983

Document Version

Publisher's PDF, also known as Version of record

Link back to DTU Orbit

Citation (APA):

Guldbrandsen, T., Guldbrandsen, B., Warner, F. L., Herman, P., \& Parkes, G. W. . (1983). Experimental Test of a New Precision Model for Microwave Rotary Vane Attenuators. I E E E Transactions on Instrumentation and Measurement, 32(1), 289-291. https://doi.org/10.1109/TIM.1983.4315061

\section{General rights}

Copyright and moral rights for the publications made accessible in the public portal are retained by the authors and/or other copyright owners and it is a condition of accessing publications that users recognise and abide by the legal requirements associated with these rights.

- Users may download and print one copy of any publication from the public portal for the purpose of private study or research.

- You may not further distribute the material or use it for any profit-making activity or commercial gain

- You may freely distribute the URL identifying the publication in the public portal 


\title{
Experimental Test of a New Precision Model for Microwave Rotary Vane Attenuators
}

\author{
TOM GULDBRANDSEN, BIRTHE GULDBRANDSEN, FRANK L. WARNER, PETER HERMAN, AND \\ GORDON W. PARKES
}

\begin{abstract}
Detailed measurements of scattering parameters have been made on a precision rotary vane attenuator (RVA) with highresolution, direct angular readout. The aim of the measurements has been to test if the RVA can be used as a primary standard of attenuation by taking advantage of a precision model described recently by one of the authors (T. Guldbrandsen). Precision attenuation measurements have been made versus angle of rotation by means of a modulated subcarrier system. Within the uncertainty, the attenuation measurements agree with the functional dependence predicted by the model. Reflection coefficients have been measured versus angle of rotation by means of a computer-corrected automatic network analyzer and, within the uncertainty, they agree with the model. From the reflection measurements, corrections to the attenuation were calculated using relations derived from the model. The corrections were compared with the measured attenuation values. So far the agreement is not quite satisfactory and additional diagnostic measurements are planned.
\end{abstract}

\section{INTRODUCTION}

$\mathrm{E}$ VER SINCE its invention more than three decades ago, the Rotary Vane Attenuator (RVA) has been a popular type of microwave attenuator. This popularity arises from its high resolution and resettability, and from the simple relationship between the attenuation and the angle of rotation, a relationship that is fulfilled with satsifactory accuracy for many purposes.

For precision applications, special laboratory RVA's have been made featuring rugged mechanical construction, highresolution readout, and smooth transitions in order to reduce mismatch, etc. A few RVA's in the world are fitted with precision, direct angular readout in order to remove mechanical errors of which especially gearing errors would otherwise cause serious problems [1], [2].

Even these precision RVA's, with direct angular readout, show systematical errors of electromagnetic origin that are considerably larger than the accuracy of the best methods for attenuation measurements. On the other hand, precision RVA's show a resettability and a stability that is at least as good as the best methods for measurements of attenuation. On this basis, it seems likely that precision RVA's could be used as primary standards of attenuation if the systematic errors could be removed in some way. As it is considered too difficult to reduce the mismatch to a tolerable level, an attempt has

Manuscript received July $15,1982$.

T. Guldbrandsen and B. Guldbrandsen are with the Physics Laboratory III, The Technical University of Denmark, 2800 Lyngby, Denmark.

F. L. Warner, P. Herman, and G. W. Parkes are with the Royal Signals and Radar Establishment, Great Malvern, Worcs., England.

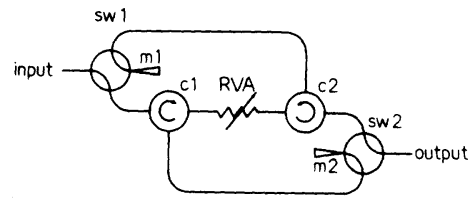

Fig. 1. RVA in circuit with circulators $c_{1}, c_{2}$, switches $s w 1, s w 2$, and matched terminations $m 1$ and $m 2$. Circuit shown in position for measurements of "forward" transmission. Reflection is measured if one of the switches is turned. If both switches are turned, the circuit is in position for measurements of "reverse" transmission.

instead been made to include all electromagnetic errors in a precision model [3].

The functional behavior is then known and methods are derived for measurements of all the parameters that are needed for a complete determination of the complex transmission versus angle of rotation. At low-attenuation settings, errors caused by internal mismatch dominate and typically amount to $0.01 \mathrm{~dB}$ for a precision RVA. In [3], it has been shown that these errors can be calculated from measurements of complex reflection coefficients versus angle.

The aim of this paper is to experimentally investigate how well the model agrees with detailed precision measurements, and thereby discuss whether or not the RVA can be used as a primary standard.

\section{METHODS FOR MEASUREMENT}

All measurements were made on an $X$-band precision RVA labeled $X R 1$. A detailed description of $X R 1$ is given in [2]. In order to enable in situ measurements, the RVA was fitted with circulators and switches as shown in Fig. 1 and described in detail in [3].

\section{Reflection Coefficients}

These were measured by means of a computer-corrected automatic network analyzer. For each frequency, the complex reflection coefficients were measured for both ports at every $15^{\circ}$ from $0^{\circ}$ to $165^{\circ}$ with a resolution of $10^{-4}$ on magnitude and $0.1^{\circ}$ on phase angle. The magnitudes are of the order of 0.05 so that the resolution is roughly $10^{-4}$ on both the real and the imaginary part.

\section{Complex Transmission Coefficients}

The complex transmission coefficients "forward" and "reverse" were measured at $0^{\circ}$ by means of the network analyzer 


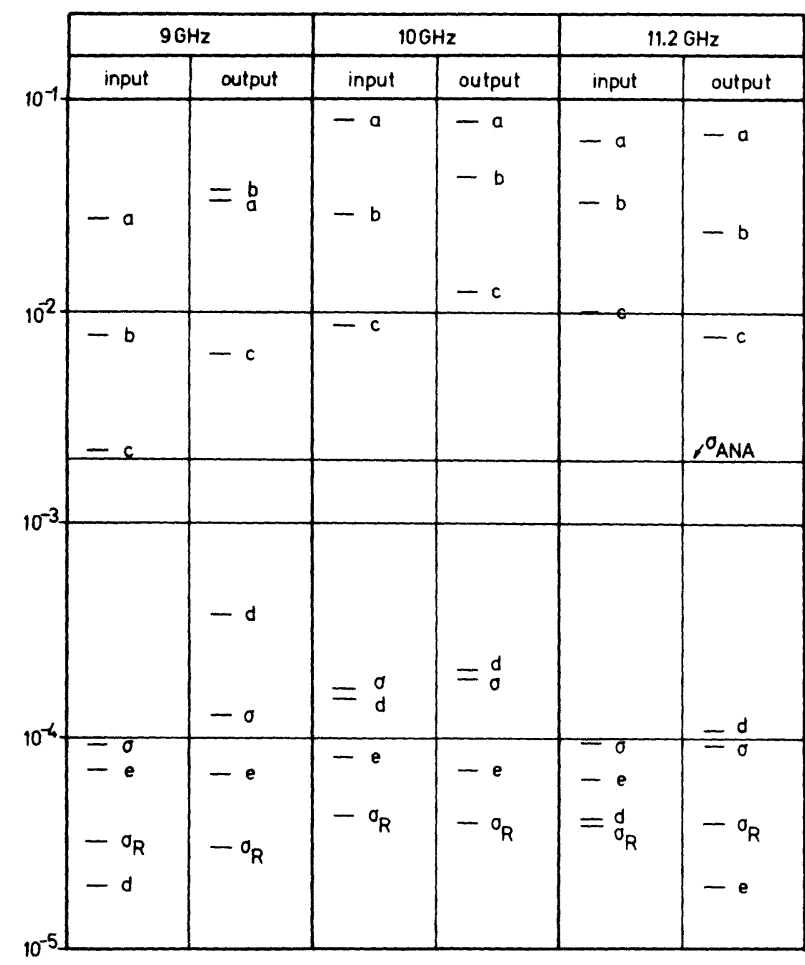

Fig. 2. Results of network analyzer measurements of reflection parameters $a, b, c, d$, and $e$. In addition, the uncertainty $\sigma$ calculated from the rms value of the unexpected Fourier coefficients, the uncertainty $\sigma_{R}$ caused by limited resolution, and, finally, the uncertainty $\sigma_{A N A}$ expected from the network analyzer specifications are shown.

as these are also required for the calculations of the transmission error parameters.

\section{Precision Attenuation Measurements}

Precision attenuation measurements were made using a modulated subcarrier system described in [4]. For each of the possible frequencies $(9,10$, and $11.2 \mathrm{GHz})$, the attenuation was measured at angles $0^{\circ}, 18.4^{\circ}, 26.6^{\circ}, 33.2^{\circ}, 39.2^{\circ}, 45.0^{\circ}$, $50.8^{\circ}, 56.8^{\circ}, 63.4^{\circ}$, and $71.6^{\circ}$ corresponding approximately to the equidistant series of transmission factors: $1,0.9, \cdots, 0.1$. Rounded angle values were chosen in order to avoid cyclic interpolation errors. The resolution of the modulated subcarrier measurements is $10^{-5}$ times the input signal corresponding to $10^{-4}-10^{-3} \mathrm{~dB}$ on attenuation values from 0 to $20 \mathrm{~dB}$.

\section{RESULTS}

From the measured reflection coefficients at the set of 12 equidistant angles, 12 Fourier components are calculated. The wanted model parameters $a, b, c, d$, and $e$ are calculated from the first five Fourier components, i.e., the dc component and the sine and cosine components of the second and fourth harmonics in the angle of rotation. Magnitudes of these parameters are presented on Fig. 2. The residual seven Fourier components, which are not present in the model, are all small and are considered to be due to uncertainty. On the basis of the seven Fourier components, the uncertainty $\sigma$ is calculated. For comparison, the uncertainty determined by the limited resolution $\sigma_{R}$ is also shown. The maximum error on measured small reflection coefficients is estimated to be $2 \times 10^{-3}$ [5].

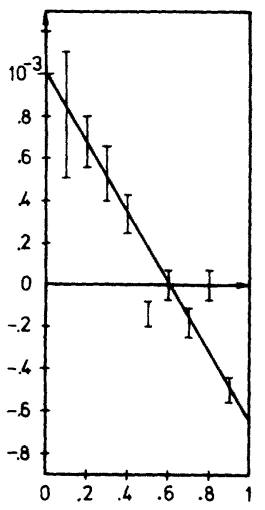

(a)

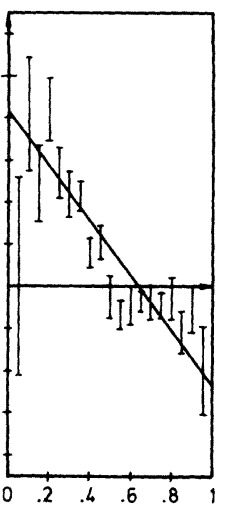

(b)

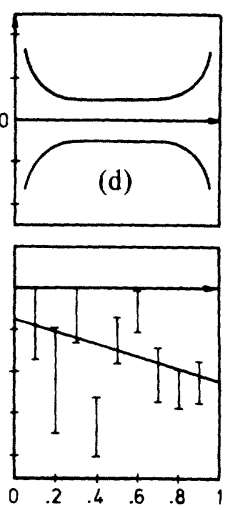

(c)
Fig. 3. Results of modulated subcarrier attenuation measurements at (a) 9 , (b) 10 , and (c) $11.2 \mathrm{GHz}$. Error bars correspond to the uncertainty determined from repeated measurements. (d) The uncertainty expected from the combined effect of $\pm 0.001^{\circ}$ angle resolution and $\pm 10^{-5} \times$ input signal (signal resolution). Vertical scale: $\left(T(\theta)-T(0) \cos ^{2} \theta\right) /\left(\sin ^{2} \theta \cos ^{2} \theta\right)$; horizontal scale: $\sin ^{2} \theta$.

From Fig. 2 we can conclude that

1) the parameters, $a, b$, and $c$ are strongly significant,

2) $d$ is barely significant and $e$ is not,

3) the agreement with the model is excellent $(\sigma \leq 2 \times$ $\left.10^{-4}\right)$.

\section{Precision Attenuation Measurements}

In contrast to the case of reflection measurements, the uncertainty of the attenuation measurements depends strongly on the vane angle. Therefore, Fourier analysis is not as straightforward to apply in attenuation measurements. Instead, the results are analyzed graphically. As the maximum attenuation is only $20 \mathrm{~dB}$, contributions from zero-setting error parameter $\theta_{0}$ and residual transmission $\gamma$ are small. These error parameters are determined from attenuation measurements around $90^{\circ}$ and all the results presented are corrected for these errors. In Fig. 3(a)-(c), the results of the precision attenuation measurements are presented.

Although the deviations between the measurements and the theoretical expression for the attenuation $40 \log \cos \theta$ are small, there are significant error contributions of the kinds described by the parameters $\operatorname{Re}(\alpha)$ and $\operatorname{Re}(\beta)$. Moreover, when $\operatorname{Re}(\alpha)$ and $\operatorname{Re}(\beta)$ have been fitted there is hardly any statistically significant systematic error left.

\section{COMPARISON OF MEASURED AND CALCUlATED ATTENUATION ERRORS}

In Fig. 4(a)-(f), the values of $\alpha$ and $\beta$ calculated from the measured values of $b$ and $c$ are shown together with the directly measured values of $\operatorname{Re}(\alpha)$ and $\operatorname{Re}(\beta)$. In spite of the good agreement shown for both the reflection and the attenuation measurements, these results show a complete disagreement.

\section{DISCUSSION}

The most obvious explanation for the discrepancy between measured and calculated attenuation errors in spite of the otherwise good agreement with the model would be that the model is correct whereas the relations used for calculation of 


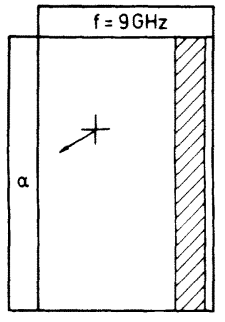

(a)

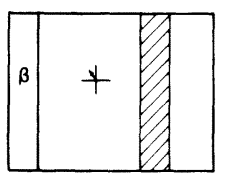

(d)

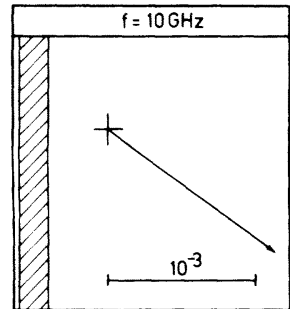

(b)

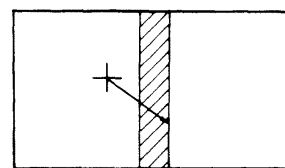

(e)

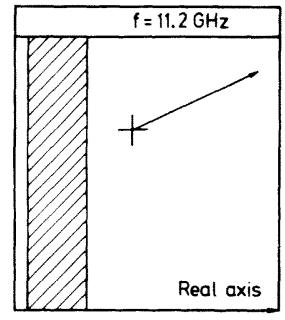

(c)

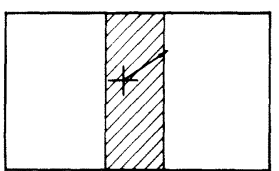

(f)
Fig. 4. Comparison of transmission error parameters. Crosses represent origins. Hatched bands represent attenuation parameters $\operatorname{Re}(\alpha)$ and $\operatorname{Re}(\beta)$ with estimated uncertainty intervals. $\operatorname{Re}(\alpha)$ and $\operatorname{Re}(\beta)$ are determined from straight-line fits to the modulated subcarrier measurements in Fig. 3 Vectors represent complex $\alpha$ and $\beta$ values calculated from reflection measurements. The uncertainty of the latter is negligible on the figure.

attenuation errors are wrong. We doubt that this is the case, because the relations have been checked several times by other researchers in independent ways.

Another possibility is that mechanical errors play a role. It is, nevertheless, unlikely that mechanical errors could have this specific angular dependence. The measured frequency dependence is also difficult to relate to mechanical errors. Finally, special high-quality bearings, etc., are used and readout errors are negligible.

An explanation that cannot be rejected is that modes other than the fundamental $\left(\mathrm{TE}_{11}\right)$ are significant at the rotary joints. It is already known that $\mathrm{TE}_{21}$ modes can cause large errors because of resonance effects, but the highest frequency used $(11.2 \mathrm{GHz})$ is below cutoff for $\mathrm{TE}_{21}$. These modes can, nevertheless, be locally present at the rotary joints. It is quite likely that such nonpropagating modes give contributions to the transmission that depend on vane angle in the same way as do the $\alpha$ - and $\beta$-type errors.

This problem can be eliminated by moving the fixed and rotary vanes away from the rotary joints and by machining the waveguides adjacent to the rotary joints with the highest possible precision.

\section{CONCLUSION}

The good agreement between the angular dependence predicted by the model and the measurements looks promising for using the RVA as a primary standard of attenuation. However, the missing fulfillment of the relationship between transmission and reflection coefficients is unsatisfactory and further theoretical as well as experimental work has to be done before a final decision can be reached.

\section{APPENDIX}

\section{MAIN RESUltS OF THE MODEL FOR RVA}

The actual circuit is shown in Fig. 1. The reflection coefficient from the input port (index label $R$ )

$$
\rho_{R}=a_{R}+b_{R} \sin ^{2} \theta+c_{R} \sin ^{2} 2 \theta+d_{R} \sin 2 \theta+e_{R} \sin 4 \theta
$$

where $a, b, c, d$, and $e$ are complex error parameters and $\theta$ is the vane angle. A similar expression is obtained for the output port by replacing index $R$ with $T$.

The transmission coefficient from the input to the output ports (forward)

$$
\begin{aligned}
T_{f}= & T_{F} \cos ^{2} \theta\left(1+\alpha \sin ^{2} \theta+\beta \sin ^{2} 2 \theta+\left(2 \theta_{0}+j \epsilon\right) \tan \theta\right. \\
& \left.+\gamma \tan ^{2} \theta+\zeta \sin 2 \theta+\eta \sin 4 \theta\right)
\end{aligned}
$$

where $T_{F}$ is the transmission coefficient for $\theta=0$ and $\alpha, \beta, \gamma$, $\zeta$, and $\eta$ are complex error parameters. $\theta_{0}$ is the zero-setting error (real) and $\epsilon$ is a real error parameter. A similar expression for the reverse transmission is obtained by replacing indexes $f$ and $F$ with $r$ and $R$.

$\alpha$ and $\beta$ can be calculated from $b_{R}, b_{T}, c_{R}$, and $c_{T}$ using the relations

$$
\begin{aligned}
& \alpha=-\frac{4}{T_{F} T_{R}}\left(b_{R} c_{T}+b_{T} c_{R}-4 c_{R} c_{T}\right) \\
& \beta=-\frac{4}{T_{F} T_{R}} c_{R} c_{T} .
\end{aligned}
$$

Similar relations for $\zeta$ and $\eta$ are not presented as these parameters are insignificant in the present case.

\section{REFERENCES}

[1] W. E. Little, W. Larson, and B. J. Kinder, "Rotary vane attenuator with an optical readout," J. Res. Nat. Bur. Stand., vol. 75C, pp. 1-5, 1971.

[2] F. L. Warner, D. O. Watton, and P. Herman, "A very accurate X-band rotary vane attenuator with an absolute digital angular measuring system," IEEE Trans. Instrum. Meas., vol. IM-21, pp. 446-450, 1972.

[3] T. Guldbrandsen, "Precision model for microwave rotary vane attenuator," IEEE Trans. Instrum. Meas., vol. IM-28, pp. 59-66, 1979.

[4] F. L. Warner, Microwave Attenation Measurements. Peter Peregrinus, 1977, pp. 136-151.

[5] A. F. Adam, "A new precision automatic microwave measurement system," IEEE Trans., Instrum. Meas., vol. IM-17, pp. 308-313, 1968. 